\title{
Oxidative stress predicts cognitive decline with aging in healthy adults: an observational study
}

\author{
Ihab Hajjar*, Salim S. Hayek, Felicia C. Goldstein, Greg Martin, Dean P. Jones and Arshed Quyyumi
}

\begin{abstract}
Background: Redox signaling, which can be assessed by circulating aminothiols, reflects oxidative stress (OS) status and has been linked to clinical cardiovascular disease and its risk factors. These, in turn, are related to executive function decline. OS may precede the pro-inflammatory state seen in vascular disease. The objective of this study is to investigate the association between aminothiol markers of OS and inflammation in cognitive decline, especially in the executive cognitive domain which is highly susceptible to cardiovascular risk factors and is an important predictor of cognitive disability.

Methods: The study design is that of a longitudinal cohort study within the setting of a large academic institution with participants being university employees $(n=511)$, mean age 49 years, 68\% women, and 23\% African-American. These participants were followed for four consecutive years with a yearly cognitive assessment conducted using computerized versions of 15 cognitive tests. Peripheral cystine, glutathione, their disulfide derivatives, and C-reactive protein (CRP) were measured.

Results: Lower levels of glutathione at baseline was associated with a decline in the executive domain over 4 years (covariate-adjusted relative risk (RR) for glutathione $=1.70(95 \% \mathrm{Cl}=1.02-2.85), p=0.04)$. Furthermore, a longitudinal decline in glutathione level was associated with a faster decline in the executive domain $(p=0.03)$. None of the other OS markers or CRP were linked to cognitive decline over 4 years.

Conclusion: Increased OS reflected by decreased glutathione was associated with a decline in executive function in a healthy population. In contrast, inflammation was not linked to cognitive decline. OS may be an earlier biomarker that precedes the inflammatory phase of executive decline with aging.
\end{abstract}

Keywords: Aging, Cognition, Oxidation, Inflammation, Glutathione, Cysteine

\section{Background}

Oxidative stress (OS) that is marked by the increased production of reactive oxygen species (ROS) has been implicated in both cardiovascular pathophysiology and in neurodegeneration [1-3]. OS has also been proposed as a fundamental driver of aging where increased production of ROS and/or decreased antioxidant defenses lead to macromolecular damage, impaired organ function, and development of disease [4]. However, clinical trials with non-specific antioxidants such as vitamins have failed to demonstrate benefit. This may be related

* Correspondence: ihabhajjar@emory.edu

Emory Univeristy, 1841 Clifton Road NE, Atlanta, GA 30329, United States to the fact that a focus on ROS is simplistic, as some ROS species such as superoxide anion radical, hydrogen peroxide, and nitric oxide also play an essential role in signaling and other physiological processes in the vascular system and the brain [5]. An alternative approach of OS as a disruption of redox signaling and control has been proposed [6].

Aminothiol-containing proteins are prone to oxidationreduction reactions. Biological organization and several physiological functions are mediated by these switches in proteins [7]. In this organizational structure, glutathione functions as a major antioxidant in tissues, supporting the elimination of peroxides and detoxification of reactive aldehydes and other toxic chemicals. In the plasma, cysteine 
undergoes oxidation to its disulfide, cystine, which must be cleared from the circulation and reduced back to cysteine to prevent disruption of the thiol switching mechanisms [8]. Quantifying these circulating thiol proteins thus may provide a more accurate assessment of systemic OS allowing the investigation of its contribution to aging and chronic disease [9].

We have shown that increased OS as assessed by measuring circulating aminothiols is associated with cardiovascular risk factors, subclinical cardiovascular disease, and are strong predictors of incident adverse cardiovascular events and mortality [10-14]. Since cardiovascular risk factors are also associated with a decline in executive function, we hypothesized that aminothiol markers of OS will be predictive of cognitive decline in healthy adults without dementia $[15,16]$. To test our hypothesis, we investigated the association between aminothiol markers of OS as well as the inflammatory marker, $\mathrm{C}$-reactive protein (CRP), and cognitive decline, especially in the executive cognitive domain which is highly susceptible to cardiovascular risk factors in a healthy population free of significant cardiovascular disease or cognitive disorders.

\section{Methods}

\section{Study description}

This study was conducted in the Center for Health Discovery and Well Being at Emory University and Georgia Institute of Technology which recruited a cohort of institutional employees [17] (http://predictivehealth.emory.edu) as described previously [18]. The Emory University Institutional Review Board approved the protocols, and informed consent was obtained from all participants. Exclusion criteria were a history in the past year of hospitalization (except for accidents); severe psychosocial disorders; addition of new prescription medications to treat a chronic disease (except for changes in antihypertensive or antidiabetic agents); drug abuse or alcoholism; a current active malignant neoplasm; uncontrolled or poorly controlled autoimmune, cardiovascular, endocrine, gastrointestinal, hematologic, infectious, inflammatory, musculoskeletal, neurological, psychiatric, or respiratory disease; and any acute illness in the 2 weeks before the baseline studies. Physical measures (blood pressure, heart rate, dualenergy X-ray absorptiometry, body mass index, and treadmill testing), laboratory tests (metabolic, hematologic and inflammatory markers), cardiovascular function, health behaviors, medication profiles, mental health markers, and cognitive function were measured at yearly intervals.

\section{Cognitive assessment}

Commonly employed versions of neuropsychological measures were administered via computer at baseline and then yearly for a total of four times, using a software developed by Aharonson and colleagues [19-21]. Cognitive tests included memory delayed recall, memory recognition, visual-spatial learning, spatial short-term memory, pattern recall, delayed pattern recall and recognition of pattern, executive function test, mental flexibility, digit symbol substitution test, forward and backward digit span, symbol spotting, and focused and sustained attention (computerized score: $0-100 \%$ correct adjusted for skill levels). The symbol spotting includes a set of 20 symbols, and the subject is asked to press the "+" symbol when the plus symbol appears. Other tests, such as executive function, included identifying the odd pattern by pressing the corresponding number of the pattern out of multiple patterns. The digit symbol substitution test included showing the subject a code for symbols and digits then the subject is instructed to substitute each symbol with the appropriate digit underneath it. Digit span instructs the subject to repeat a set of digits (forward or backward) after showing them the set of digits. A full description of this battery is available here [19-21]. Cognitive scores for cognitive domains were derived using principal component analysis with Varimax (orthogonal) rotation and Kaiser normalization to perform the exploratory factor analysis and was then followed with a confirmatory factor analysis by exploring the correlations and model fit of the derived factor-saved scores as reported previously in our reports [22, 23]. The factor analysis resulted in deriving three scores related to executive function, memory, and working memory. The distributions of the cognitive scores were extremely skewed to the right, as were the raw scores, suggesting a highperformance level of the participants (probably related to the high educational levels of the sample: 18.7 (standard error $=0.2$ years) and a ceiling effect of the tests used. Test scores were divided in all the analyses at each visit into binomial variables: low vs high performance if a participant's score was below the cut-off of the lowest quartile of the corresponding score at baseline. Factor analysis and cut-off were derived from the baseline sample of 601 participants.

\section{Markers of oxidative stress and inflammation}

Plasma cysteine (CyS), its oxidized form cystine (CySS), glutathione, and its oxidized form glutathione disulfide (GSSG) were measured by high-performance liquid chromatography $[6,9]$. Blood samples were drawn and transferred into pre-prepared Eppendorf tubes containing preservatives to retard auto-oxidation, centrifuged, and stored at $-80{ }^{\circ} \mathrm{C}$ for no more than 2 months. Sample collection and storage conditions have been previously described [24]. Analyses by high-performance liquid chromatography were performed after dansyl derivatization on a 3-aminopropyl column with fluorescence detection. Metabolites were identified by coelution with standards and quantified by integration relative to the internal standard, with validation relative to external standards. Higher levels of the oxidized derivatives and lower levels of the reduced forms represent increased OS. 
The coefficient of variation for cystine is $3.2 \%$ and glutathione $5 \%$. Subjects also underwent venous blood collection for the measurement of the inflammatory marker, CRP via Multiplex kit (R\&D Systems, Minneapolis, MN).

\section{Statistical analysis}

We limited our analysis to those with both cognitive and OS and inflammatory cytokines assessments at baseline who had two separate cognitive assessments during follow-up (n =511). To estimate the age, gender, and race-adjusted change in the calculated cognitive scores, we performed multiple regression analysis after adjusting for these three demographic variables. We provide the rate as a unit of change in the calculated score per year (unit/year). Baseline correlations between OS markers and cognitive scores were performed using regression analyses. Cognitive scores and aminothiol levels were included as discrete variables due to their skewness in the longitudinal analysis. We used binomial regression with generalized estimating equations (GEE) for repeated discrete measures. GEE is appropriate for binary repeated correlated measures, and it allows for the estimation of risk in the population $[25,26]$. The cumulative relative risk of having cognitive decline (below the cut-off derived from the baseline score) was calculated during the follow-up [27]. To assess the relation between changes in OS and cognition, we first calculated a within-subject slope for each measure (yearly change) and then performed multiple standardized regressions between the rate of change in OS markers and cognitive measures. All analysis models were adjusted for age, gender, race, education, systolic blood pressure, statin, and antihypertensive therapy. All analyses were conducted in SAS (V9.4, Carey, NC).

\section{Results}

Our final analytical sample was 511 (85\% of the 601 who had cognitive assessment at baseline). The mean age at baseline was 48 years, $68 \%$ were women and $24 \%$ were AfricanAmerican, Table 1 . The median number of follow-up visits with OS and cognitive assessments was 4, and the mean follow-up time was 49.4 months. Pearson correlation coefficients between CRP and OS markers were -0.07 with glutathione $(p=0.07),-0.008$ with GSSG $(p=0.84), 0.06$ with CYS $(p=0.18)$, and 0.18 with CYSS $(p<0.001)$. Over the study period, the age-, gender-, race-adjusted decline in the executive domain was 0.25 unit/year (95\% CI $0.21-0.30$ ), in the memory domain was 0.23 unit/ year (95\% CI 0.19-0.27), and in the working memory domain was 0.23 unit/year (95\% CI 0.19-0.28).

Baseline association between OS and cognitive performance: At baseline, higher GSSG levels, indicative of higher OS, were associated with worse performance on the memory $(p=0.009)$ and working memory $(p<0.0001)$ domains but with better performance on the executive function domain $(p<0.0001)$. None of the other markers were
Table 1 Baseline characteristics $(n=511)$ of the participants with OS markers and at least two cognitive evaluations

\begin{tabular}{|c|c|c|}
\hline & Mean or count & Standard error or $\%$ \\
\hline Age, years & 49.1 & 0.5 \\
\hline Women & 348 & $68 \%$ \\
\hline Race: White/African-American & $366 / 117$ & $72 \% / 23 \%$ \\
\hline Body mass index, $\mathrm{kg} / \mathrm{m}^{2}$ & 27.68 & 0.27 \\
\hline Years of education & 18.91 & 0.2 \\
\hline Systolic blood pressure, $\mathrm{mmHg}$ & 121 & 1 \\
\hline Diastolic blood pressure, $\mathrm{mmHg}$ & 76 & 0.5 \\
\hline Hypertension & 156 & $31 \%$ \\
\hline Hypercholesterolemia & 96 & $19 \%$ \\
\hline Diabetes mellitus, type 2 & 20 & $4 \%$ \\
\hline CRP, mg/L & 0.2 & 0.02 \\
\hline \multicolumn{3}{|l|}{ Markers of oxidative stress } \\
\hline $\mathrm{GSH}, \mu \mathrm{mol} / \mathrm{L}$ & 4.4 & 0.1 \\
\hline GSSG, $\mu \mathrm{mol} / \mathrm{L}$ & 0.06 & 0.01 \\
\hline CYS, $\mu \mathrm{mol} / \mathrm{L}$ & 9.3 & 0.09 \\
\hline Cystine, $\mu \mathrm{mol} / \mathrm{L}$ & 85.3 & 0.8 \\
\hline \multicolumn{3}{|l|}{ Cognitive function score } \\
\hline Memory domain & 65.7 & 0.7 \\
\hline Executive function domain & 83.6 & 0.2 \\
\hline Working memory domain & 55.0 & 0.4 \\
\hline
\end{tabular}

Cys cysteine, CYSS cystine, GSH glutathione, GSSG glutathione disulfide

Table 2 Baseline cross-sectional associations between cognitive scores and OS markers/CRP

\begin{tabular}{llllll}
\hline Cognitive domain & Marker & Unadjusted & & Multivariate & $p$ value \\
\hline Executive domain & & & & $\beta$ & \\
& CRP & 0.24 & 0.63 & 0.109 & 0.86 \\
& CyS & -0.088 & 0.27 & -0.11 & 0.24 \\
& CySS & -0.013 & 0.16 & -0.0028 & 0.81 \\
& GSSG & 16.35 & $<.0001$ & 17.91 & $<.0001$ \\
& GSH & 0.18 & 0.12 & 0.23 & 0.095 \\
Memory domain & & & & & \\
& CRP & 0.14 & 0.9 & 0.19 & 0.88 \\
& CyS & 0.13 & 0.46 & 0.29 & 0.14 \\
& CySS & -0.0047 & 0.82 & 0.013 & 0.63 \\
& GSSG & -19.17 & 0.009 & -22.25 & 0.0035 \\
& GSH & 0.020 & 0.94 & -0.048 & 0.877 \\
Working memory domain & & & & \\
& CRP & -2.27 & 0.0028 & -1.67 & 0.063 \\
& CyS & -0.20 & 0.089 & -0.19 & 0.14 \\
& CySS & -0.0079 & 0.57 & 0.0091 & 0.60 \\
GSSG & -30.78 & $<.0001$ & -33.67 & $<.0001$ \\
GSH & -0.22 & 0.22 & -0.47 & 0.020 \\
\hline
\end{tabular}

All models were adjusted for race, age, gender, systolic blood pressure, body mass index, education, statin, and antihypertension medication $\beta$ is derived from the regression analyses of the oxidative stress marker vs cognitive function

CRP C-reactive protein, Cys cysteine, CYSS cystine, GSH glutathione, GSSG glutathione disulfide 


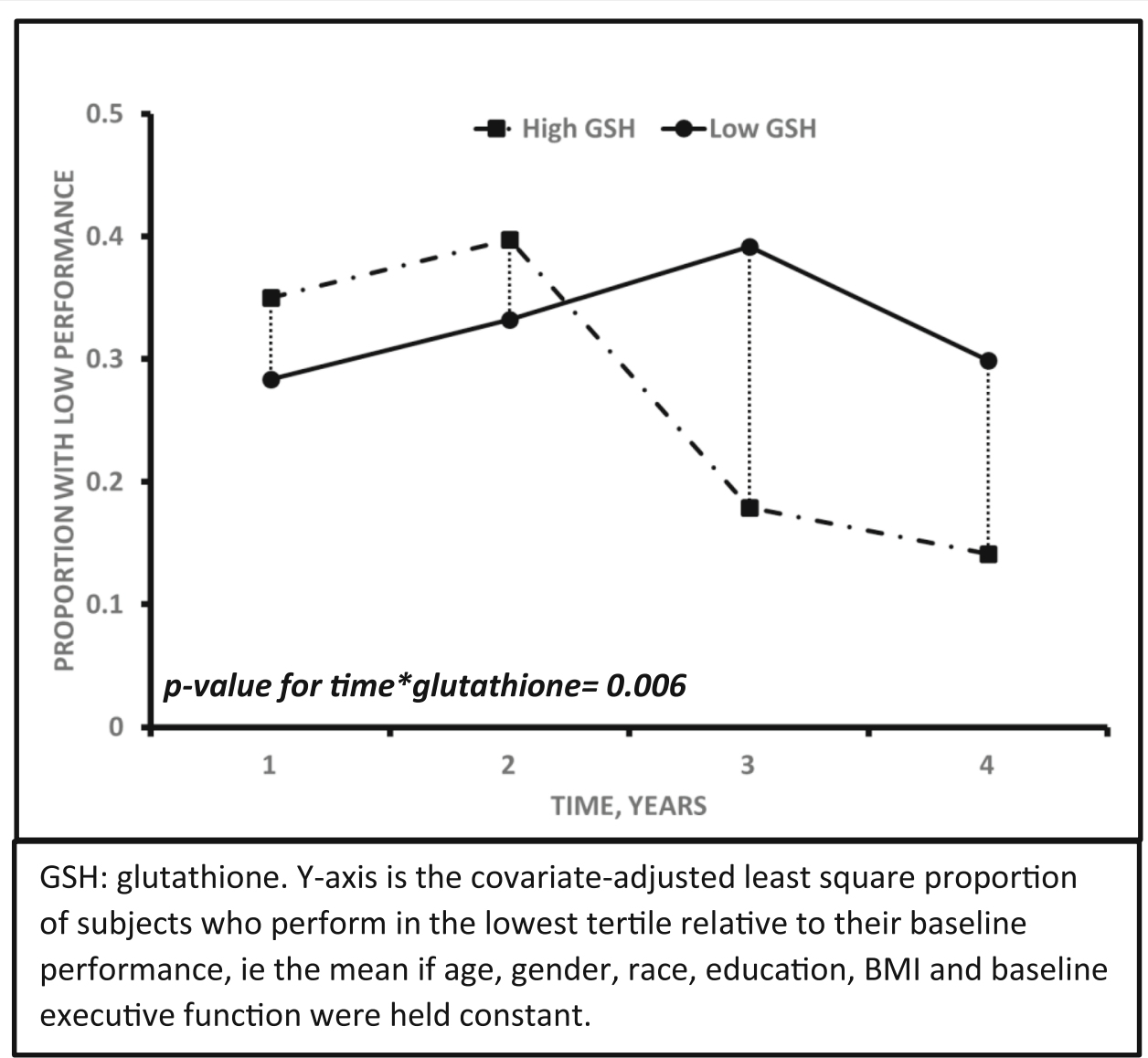

Fig. 1 Association of baseline glutathione with the proportion of subjects with decreased executive function over the 4-year period

associated with baseline memory domains (Table 2). CRP tended to be associated with baseline working memory $(p=0.06)$ but not memory or executive function.

Relationship between baseline OS and longitudinal cognitive decline: Lower levels of glutathione at baseline, reflecting increased OS, were associated with a greater likelihood of having a lower score on the executive domain over 4 years [covariate-adjusted relative risk $(\mathrm{RR})=1.70(95 \% \mathrm{CI}=1.02-2.85), p=0.04]$. In those with high (>1 SD units) glutathione levels at baseline, the number of participants performing below the cut-off (bottom quartile score at baseline) for the executive domain decreased from $35 \%$ at baseline to $14 \%$ at year 4 . In contrast, that number increased from 28 to $30 \%$ in those with low glutathione levels at baseline. The difference between the baseline high vs low glutathione levels was statistically significant ( $p$ value for time*baseline glutathione $=0.006$ ) as shown in Fig. 1. Baseline CRP, CYS, CYSS, and GSSG levels were not associated with the change in executive function or other memory domains.

Change in markers of oxidative stress and cognitive decline: Longitudinal decreases in glutathione level $(\beta=$ $-1.7, p=0.03)$ were associated with a greater decline in the executive domain, after adjusting for age, gender, race, education, blood pressure, use of statin, and antihypertensive therapy (Table 3). For every unit decline in glutathione, executive function declined by 1.7 units $(p=0.03)$, and this was not true for any other OS marker.

\section{Discussion}

This study demonstrates that decreased circulating levels of glutathione predict the age-related decline in the executive domain, an area of cognition that is particularly susceptible to cardiovascular disease. In addition, higher levels of the oxidized glutathione, GSSH, are related to lower performance on both memory and working memory but better executive function. Most uniquely in our analysis, we demonstrate that age (or time)-related changes in OS correlate with the age-related decline in the executive domain. Extending our previous reports relating aminothiol levels with subclinical vascular disease and incident cardiovascular events, we now demonstrate that circulating aminothiol levels serve as a biomarker of change in executive function [11].

The existence of a role for OS in vascular disease and cognitive aging has been suggested, but most studies have so far focused on ROS [28]. Our findings indicate that 
Table 3 Unadjusted and adjusted population risk for having cognitive decline to below the baseline cut-off for the corresponding cognitive domain during the study visits [1-4]

\begin{tabular}{|c|c|c|c|c|c|c|c|c|}
\hline & \multicolumn{3}{|c|}{ Univariate } & \multicolumn{5}{|c|}{ Multivariate* } \\
\hline & $\overline{\mathrm{RR}}$ & $95 \% \mathrm{Cl}$ & & $p$ value & RR & $95 \% \mathrm{C}$ & & $p$ value \\
\hline \multicolumn{9}{|l|}{ Cys } \\
\hline Memory domain & 0.54 & 0.20 & 1.46 & 0.22 & 0.61 & 0.20 & 1.87 & 0.39 \\
\hline Executive function domain & 1.11 & 0.66 & 1.86 & 0.70 & 1.08 & 0.65 & 1.78 & 0.78 \\
\hline Working memory domain & 0.68 & 0.42 & 1.08 & 0.10 & 0.56 & 0.35 & 0.90 & 0.05 \\
\hline \multicolumn{9}{|l|}{ CYSS } \\
\hline Memory domain & 0.89 & 0.50 & 1.58 & 0.69 & 0.92 & 0.51 & 1.66 & 0.78 \\
\hline Executive function domain & 0.96 & 0.60 & 1.52 & 0.86 & 0.82 & 0.51 & 1.31 & 0.41 \\
\hline Working memory domain & 1.15 & 0.72 & 1.85 & 0.56 & 1.07 & 0.65 & 1.76 & 0.78 \\
\hline \multicolumn{9}{|l|}{ GSH } \\
\hline Memory domain & 0.83 & 0.43 & 1.58 & 0.57 & 0.64 & 0.31 & 1.32 & 0.23 \\
\hline Executive function domain & 1.82 & 1.10 & 3.01 & 0.02 & 1.70 & 1.02 & 2.85 & 0.04 \\
\hline Working memory domain & 0.97 & 0.60 & 1.57 & 0.91 & 1.08 & 0.63 & 1.86 & 0.78 \\
\hline \multicolumn{9}{|l|}{ GSSG } \\
\hline Memory domain & 1.15 & 0.72 & 1.85 & 0.56 & 1.07 & 0.65 & 1.76 & 0.78 \\
\hline Executive function domain & 1.18 & 0.65 & 2.15 & 0.59 & 1.56 & 0.81 & 3.04 & 0.19 \\
\hline Working memory domain & 0.83 & 0.43 & 1.58 & 0.57 & 0.64 & 0.31 & 1.32 & 0.23 \\
\hline \multicolumn{9}{|l|}{ CRP } \\
\hline Memory domain & 1.43 & 0.61 & 3.34 & 0.41 & 0.94 & 0.59 & 1.50 & 0.94 \\
\hline Executive function domain & 1.13 & 0.63 & 2.05 & 0.68 & 0.84 & 0.45 & 1.54 & 0.84 \\
\hline Working memory domain & 1.05 & 0.67 & 1.64 & 0.83 & 0.94 & 0.59 & 1.50 & 0.94 \\
\hline
\end{tabular}

*Models adjusted for race, age, gender, systolic blood pressure, body mass index, education, statin, and antihypertension medication use and baseline performance CRP C-reactive protein, CYS cysteine, CYSS cystine, GSH glutathione, GSSG glutathione disulfide

glutathione may predict aging-related decline in executive function. This is important because decline in executive function is a better predictor, more so than memory, of future functional loss in normal controls and those with mild cognitive impairment or dementia.

Use of free radical-scavenging antioxidants such as vitamin $\mathrm{E}$ has failed to show a consistent benefit on cognitive preservation and has been linked to increased cardiovascular mortality [29, 30]. However, vitamin E supplementation can lower glutathione levels through its effect on the glutathione S-transferase and may potentially exacerbate OS [31]. Our results demonstrating the link between aminothiol markers and cognitive preservation may offer an explanation for the inconsistent findings with vitamin $\mathrm{E}$ supplementation trials on cognitive outcomes.

Increased protein oxidation has been observed in the brains of persons with Alzheimer's disease, especially in regions that are rich in amyloid peptide [32]. The underlying mechanisms remain unknown, although increased OS is thought to be an initial trigger [33]. Glutathione plays a key role in the antioxidant defense of neuronal cells, and circulating glutathione levels are reduced in AD [34]. Our findings show that the plasma glutathione levels predict future decline in cognition. Moreover, individuals who experience decreases in glutathione level over time also have a greater decline in their cognition. These findings potentially offer new targets for the prevention and treatment of cognitive loss with aging, especially that related to executive function where no therapy is currently available.

In the brain, glutathione synthesis varies by cell type and depends on its ability to use available extracellular glutathione precursors. Neurons rely on the presence of extracellular cysteine as a precursor for glutathione [35]. Glutathione is also released from astrocytes [36-40] and can be imported from blood into the brain through the blood-brain barrier [41, 42] In particular, a sodiumdependent glutathione transporter has been identified in the brain capillaries [43] and brain endothelial cells [44]. In disease states, a reduction in brain glutathione occurs with aging, Parkinson's disease [45, 46], and Alzheimer's disease [47]. This provides a biological explanation for our observations of the association between glutathione and cognition.

We did not demonstrate an association between CRP and cognitive decline in this population, apart from the baseline association with working memory performance where the association was borderline significant. Increased CRP, a marker of increased systemic inflammation, may 
occur later in the evolution of cognitive decline with aging and in neurodegeneration [48]. Further, peripheral CRP is a surrogate marker but not causative inflammatory mediator, thus we cannot exclude the possibility that decreased serum levels of glutathione are mirrored by simultaneous increased levels of inflammatory mediators in the CNS which in turn are linked to cognitive decline.

Strengths of our study are the inclusion of healthy disease-free middle-aged individuals, the assessment of blood aminothiols as unique markers of OS, and detailed cognitive testing. The limitation of our study is relatively short follow-up period and the lack of brain imaging. In addition, peripheral GSH/GSSG as a biomarker for oxidative stress is not as good as tissue measures and hence may be missing significant other associations with cognition. The differential effects between peripheral and tissue may explain the lack of coupling between GSH and GSSG. Finally, additional oxidative stress markers from lipid and nucleic acid were not measured.

\section{Conclusion}

OS reflected by a low or a progressive decrease in glutathione levels is associated with a decline in executive function with aging. The role of OS in cognitive decline offers further insights into the processes of cognitive aging and the link with vascular risk factors and warrants further investigation.

\section{Acknowledgements}

None.

\section{Funding}

The Predictive Health Institute is supported by Emory University and the National Center for Advancing Translational Sciences of the National Institutes of Health (UL1 TR000454). This analysis was funded by grants R01AG042127 to Ihab Hajjar. Dr. Quyyumi is supported by NIH grants 5P01HL101398-02, 1P20HL113451-01, 1R56HL126558-01, 1RF1AG051633-01, R01 NS064162-01, R01 HL89650-01, HL095479-01, 1U10HL1 10302-01, 1DP3DK094346-01, 2P01 HL086773, and American Heart Association Grant no. 0000031288. Dr. Jones is supported by NIH grants P2OHL113451, ES023485, ESO25632, ES026560, HD075784, P30ES019776, AG038746, HL095479, EY022618, HL086773, CA188038, MMH107205, HHSN272201200031C, Henry M. Jackson Foundation HT9404-13-0030, and a California Breast Cancer Research Program Grant. The authors have no disclosures.

Availability of data and materials

The data are available from the corresponding author.

\section{Authors' contributions}

$\mathrm{IH}, \mathrm{AQ}, \mathrm{SH}, \mathrm{GM}$, and DJ contributed to the study concept and design. AQ, DJ, $\mathrm{FG}$ and $\mathrm{SH}$ contributed to the acquisition of subjects and/or data. IH, AQ, FG and $\mathrm{SH}$ contributed to the analysis and interpretation of data. $\mathrm{IH}, \mathrm{AQ}, \mathrm{SH}$, GM, FG and DJ contributed to the preparation of the manuscript. All authors read and approved the final manuscript.

\section{Ethics approval and consent to participate}

This study was approved by the Emory University IRB.

\section{Consent for publication}

Not applicable.

\section{Competing interests}

The authors declare that they have no competing interests.

\section{Publisher's Note}

Springer Nature remains neutral with regard to jurisdictional claims in published maps and institutional affiliations.

Received: 6 June 2017 Accepted: 7 December 2017

Published online: 16 January 2018

\section{References}

1. Hatanaka H, Hanyu H, Hirose D, Fukusawa R, Namioka N, Iwamoto T. Peripheral oxidative stress markers in individuals with Alzheimer's disease with or without cerebrovascular disease. J Am Geriatr Soc. 2015;63:1472-4.

2. Csanyi G, Miller FJ Jr. Oxidative stress in cardiovascular disease. Int J Mol Sci. 2014;15:6002-8.

3. White A, Culmsee C, Beart P. Oxidative stress and neurodegeneration. Neurochem Int. 2013:62:521.

4. Finkel T, Holbrook NJ. Oxidants, oxidative stress and the biology of ageing. Nature. 2000:408:239-47.

5. Veal E, Day A. Hydrogen peroxide as a signaling molecule. Antioxid Redox Signal. 2011;15:147-51.

6. Jones DP. Redefining oxidative stress. Antioxid Redox Signal. 2006;8:1865-79.

7. Harris $C$, Hansen JM. Oxidative stress, thiols, and redox profiles. Methods Mol Biol. 2012:889:325-46

8. Go YM, Jones DP. Intracellular proatherogenic events and cell adhesion modulated by extracellular thiol/disulfide redox state. Circulation. 2005;111:2973-80

9. Jones DP. Radical-free biology of oxidative stress. American journal of physiology Cell physiology. 2008;295:C849-68.

10. Liang LP, Patel M. Plasma cysteine/cystine redox couple disruption in animal models of temporal lobe epilepsy. Redox Biol. 2016;9:45-9.

11. Patel RS, Ghasemzadeh N, Eapen DJ, et al. Novel biomarker of oxidative stress is associated with risk of death in patients with coronary artery disease. Circulation. 2016;133:361-9.

12. Morris $A A$, Zhao $L$, Patel RS, et al. Differences in systemic oxidative stress based on race and the metabolic syndrome: the Morehouse and Emory Team up to Eliminate Health Disparities (META-Health) study. Metab Syndr Relat Disord. 2012;10:252-9.

13. Patel RS, Al Mheid I, Morris AA, et al. Oxidative stress is associated with impaired arterial elasticity. Atherosclerosis. 2011;218:90-5.

14. Ashfaq S, Abramson JL, Jones DP, et al. Endothelial function and aminothiol biomarkers of oxidative stress in healthy adults. Hypertension. 2008;52:80-5.

15. Raz N, Rodrigue KM, Acker JD. Hypertension and the brain: vulnerability of the prefrontal regions and executive functions. Behav Neurosci. 2003;117:1169-80

16. Vasquez BP, Zakzanis KK. The neuropsychological profile of vascular cognitive impairment not demented: a meta-analysis. J Neuropsychol. 2015;9:109-36

17. Tuttolomondo A, Pecoraro R, Di Raimondo D, et al. Immune-inflammatory markers and arterial stiffness indexes in subjects with acute ischemic stroke with and without metabolic syndrome. Diabetology \& metabolic syndrome. 2014:6:28

18. Rask KJ, Brigham KL, Johns MM. Integrating comparative effectiveness research programs into predictive health: a unique role for academic health centers. Academic medicine: journal of the Association of American Medical Colleges. 2011:86:718-23.

19. Korczyn AD, Aharonson V. Computerized methods in the assessment and prediction of dementia. Curr Alzheimer Res. 2007:4:364-9.

20. Aharonson V. Halperin I, Korczyn AD. Computerized diagnosis of mild cognitive impairment. Alzheimer's and dementia: the journal of the Alzheimer's Association. 2007:3:23-7.

21. Aharonson $\mathrm{V}$, Korczyn AD. Human-computer interaction in the administration and analysis of neuropsychological tests. Comput Methods Prog Biomed. 2004;73:43-53.

22. Jackson DJ, Borgatta EF. Factor analysis and measurement in sociological research: a multi-dimensional perspective. Chapter "selecting a data analysis model for factorial ecology research". Beverly Hills, Calif:. Sage Publications, 1981.

23. Brown TA. Confirmatory factor analysis for applied research. New York: Guilford Press; 2006.

24. Jones DP. Liang Y. Measuring the poise of thiol/disulfide couples in vivo. Free Radic Biol Med. 2009;47:1329-38

25. Spiegelman D, Hertzmark E, Easy SAS. Calculations for risk or prevalence ratios and differences. Am J Epidemiol. 2005;162:199-200. 
26. Zeger SL, Liang KY. Longitudinal data analysis for discrete and continuous outcomes. Biometrics. 1986;42:121-30.

27. Greenland S. Model-based estimation of relative risks and other epidemiologic measures in studies of common outcomes and in casecontrol studies. Am J Epidemiol. 2004;160:301-5.

28. Droge W, Schipper HM. Oxidative stress and aberrant signaling in aging and cognitive decline. Aging Cell. 2007;6:361-70.

29. Lonn E, Bosch J, Yusuf S, et al. Effects of long-term vitamin E supplementation on cardiovascular events and cancer: a randomized controlled trial. JAMA. 2005;293:1338-47.

30. Corbett A, Ballard C. The value of vitamin E as a treatment for Alzheimer's disease remains unproven despite functional improvement, due to a lack of established effect on cognition or other outcomes from RCTs. Evid Based Med. 2014;19:140.

31. van Haaften Rl, Evelo CT, Haenen GR, Bast A. Alpha-tocopherol inhibits human glutathione S-transferase pi. Biochem Biophys Res Commun. 2001;280:631-3.

32. Butterfield DA, Boyd-Kimball D, Castegna A. Proteomics in Alzheimer's disease: insights into potential mechanisms of neurodegeneration. J Neurochem. 2003;86:1313-27.

33. Aksenov MY, Aksenova MV, Butterfield DA, Geddes JW, Markesbery WR. Protein oxidation in the brain in Alzheimer's disease. Neuroscience. 2001;103:373-83

34. Bermejo P, Martin-Aragon S, Benedi J, et al. Peripheral levels of glutathione and protein oxidation as markers in the development of Alzheimer's disease from mild cognitive impairment. Free Radic Res. 2008:42:162-70.

35. Dringen R, Pfeiffer B, Hamprecht B. Synthesis of the antioxidant glutathione in neurons: supply by astrocytes of CysGly as precursor for neuronal glutathione. The Journal of neuroscience: the official journal of the Society for Neuroscience. 1999;19:562-9.

36. Yudkoff $M$, Pleasure $D$, Cregar $L$, et al. Glutathione turnover in cultured astrocytes: studies with [15N]glutamate. J Neurochem. 1990;55:137-45.

37. Sagara J, Makino N, Bannai S. Glutathione efflux from cultured astrocytes. J Neurochem. 1996;66:1876-81.

38. Dringen R, Kranich O, Hamprecht B. The gamma-glutamyl transpeptidase inhibitor acivicin preserves glutathione released by astroglial cells in culture. Neurochem Res. 1997:22:727-33.

39. Hirrlinger J, Schulz JB, Dringen R. Effects of dopamine on the glutathione metabolism of cultured astroglial cells: implications for Parkinson's disease. J Neurochem. 2002;82:458-67.

40. Stewart VC, Stone R, Gegg ME, et al. Preservation of extracellular glutathione by an astrocyte derived factor with properties comparable to extracellular superoxide dismutase. J Neurochem. 2002;83:984-91.

41. Favilli F, Marraccini P, lantomasi T, Vincenzini MT. Effect of orally administered glutathione on glutathione levels in some organs of rats: role of specific transporters. Br J Nutr. 1997:78:293-300.

42. Zlokovic BV, Mackic JB, McComb JG, Weiss MH, Kaplowitz N, Kannan R. Evidence for transcapillary transport of reduced glutathione in vascular perfused guinea-pig brain. Biochem Biophys Res Commun. 1994;201:402-8.

43. Kannan R, Yi JR, Tang D, Li Y, Zlokovic BV, Kaplowitz N. Evidence for the existence of a sodium-dependent glutathione (GSH) transporter. Expression of bovine brain capillary mRNA and size fractions in Xenopus laevis oocytes and dissociation from gamma-glutamyltranspeptidase and facilitative GSH transporters. J Biol Chem. 1996;271:9754-8.

44. Kannan R, Mittur A, Bao Y, Tsuruo T, Kaplowitz NGSH. Transport in immortalized mouse brain endothelial cells: evidence for apical localization of a sodium-dependent GSH transporter. J Neurochem. 1999:73:390-9.

45. Sofic E, Lange KW, Jellinger K, Riederer P. Reduced and oxidized glutathione in the substantia nigra of patients with Parkinson's disease. Neurosci Lett. 1992;142:128-30.

46. Sian J, Dexter DT, Lees AJ, et al. Alterations in glutathione levels in Parkinson's disease and other neurodegenerative disorders affecting basal ganglia. Ann Neurol. 1994;36:348-55.

47. Gu M, Owen AD, Toffa SE, et al. Mitochondrial function, GSH and iron in neurodegeneration and Lewy body diseases. J Neurol Sci. 1998;158:24-9.

48. Daulatzai MA. Fundamental role of pan-inflammation and oxidativenitrosative pathways in neuropathogenesis of Alzheimer's disease in focal cerebral ischemic rats. Am J Neurodegener Dis. 2016;5:102-30.

\section{Submit your next manuscript to BioMed Central and we will help you at every step:}

- We accept pre-submission inquiries

- Our selector tool helps you to find the most relevant journal

- We provide round the clock customer support

- Convenient online submission

- Thorough peer review

- Inclusion in PubMed and all major indexing services

- Maximum visibility for your research

Submit your manuscript at www.biomedcentral.com/submit
Biomed Central 